\title{
Visceral Microaneurysms of Polyarteritis Nodosa: Presentation with Recurrent Upper GI Haemorrhage needing Total Gastrectomy
}

\author{
Dr Senthil Kumar MP MBBS, MS, FRCS (Edin), FRCS (Gen Surg) \\ Additional Professor, Liver transplantation, ILBS, New Delhi, India \\ Mr Dipankar Mukherjee MS FRCS (Glas), FRCS (Eng), FRCS (Gen Surg) \\ Consultant Upper GI surgeon, Queens Hospital Romford, Essex, UK
}

\begin{abstract}
:
Polyarteritis nodosa (PAN) is a systemic necrotising vasculitis which is a rare but important cause of gastrointestinal bleeding. A sixty nine year old lady presented with life threatening upper gastrointestinal bleeding from the stomach. Multiple attempts at endoscopic localisation and therapy failed, as did one attempt at therapeutic splenic artery embolisation and two laparotomies. She finally underwent total gastrectomy. Gastric histology revealed necrotising arteritis consistent with polyarteritis nodosa. On retrospect she was found to have a number of features of polyarteritis nodosa, including the classic visceral microaneurysms on angiography. She responded to intravenous corticosteroids, but succumbed to a myocardial infarction three months later.
\end{abstract}

Keywords: Polyarteritis nodosa; PAN; GI haemorrhage; Gastrectomy; Microaneurysms

\section{INTRODUCTION}

Although upper gastrointestinal haemorrhage is a commonly encountered clinical problem, it is a rare initial mode of presentation for a vasculitic process such as polyarteritis nodosa (PAN). Polyarteritis nodosa is a systemic necrotising vasculitis involving the medium and small sized arteries in many vascular beds.

Though expansile arterial lesions (microaneurysms and ectasias) at arterial branch points are classical, occlusive arterial lesions may be at least equally and possibly more common. ${ }^{1}$ Gastrointestinal involvement includes bleeding, ulceration, infarction and perforation of hollow viscera including appendix and gall bladder. Abdominal pain, weight loss and malabsorption resulting in diarrhoea are well recognised presentations ${ }^{2}$. There are only a few case reports of the acute surgical complications due to PAN. However, life threatening GI haemorrhage may be the first presentation as exemplified by the patient presented herein.

\section{CASE REPORT}

A 69 year old obese, diabetic lady with a history of three prior myocardial infarctions (aged 40, 48 and 50 years respectively) and a coronary artery bypass grafting 18 years previously, presented initially with a few days history small volume coffee ground vomiting and upper abdominal discomfort. She also admitted to a six month history of intermittent non-bloody diarrhoea and fatigue. She was not on steroids or non-steroidal anti-inflammatory agents. There was no past history of peptic ulcer, cirrhosis or overt gastrointestinal bleeding. She was anaemic with haemoglobin of $7.5 \mathrm{gm} \%$. Blood platelets, coagulation, renal and hepatic parameters where within normal limits

Initial upper GI endoscopy showed minimal oesophagitis, while the stomach and duodenum were normal. As she was haemodynamically stable, she was discharged on a proton pump inhibitor after blood transfusions, with plans for outpatient investigations and follow up.

However, she presented a week later with frank haematemesis and melena leading to shock. On endoscopy, fresh blood was present in the stomach and an adherent clot in the fundus, over a possible superficial ulcer. This area was injected with adrenaline followed by tranexamic acid and parenteral 
Senthilkumar \& Dipankar Mukherjee, "Visceral Microaneurysms of Polyarteritis Nodosa: Presentation with Recurrent Upper GI Haemorrhage needing Total Gastrectomy"

omeprazole. Her melena persisted requiring multiple transfusions. A repeat endoscopy after 3 days, showed a Dieulafoy lesion in the fundus of stomach with adherent clot (fig 1).

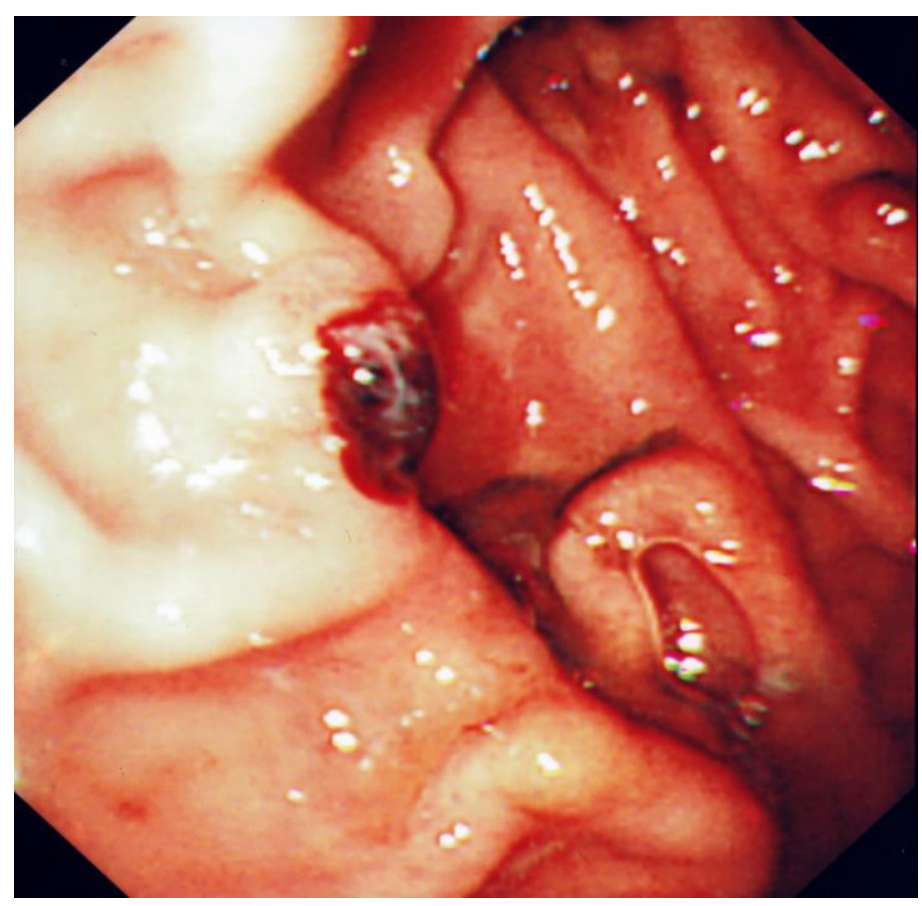

Fig1. Endoscopic view of Dieulafoy lesion in the gastric fundus

Peri-lesional adrenaline injection was carried out. This stabilised her temporarily. Seventy two hours later, she had further hematemesis and melena with episodes of hypotension which was only transiently corrected by transfusions. As endoscopy failed to provide good views in the setting of ongoing bleeding, she underwent a laparotomy.

Anterior gastrotomy, showed blood clots in the fundus, but no active bleeding. The area of bruising in the fundus and superficial ulceration, which was the only abnormality seen on per-operative endoscopy was oversewn. Forty eight hours later, she became hemodynamically unstable with a precipitous drop in haemoglobin, necessitating a further laparotomy. While there were blood clots in the stomach, there was no active bleeding. No further surgical procedure was done. An immediate post-operative endoscopy showed fundal mucosal edema, but no bleeding.

She continued to have intermittent bleeding as evidenced by coffee ground nasogastric aspirate and melena requiring transfusion. As she became hemodynamically unstable once more, it was decided to perform a celiac axis angiogram with a view to possible embolisation. The angiogram showed a blush of contrast along the greater curve of the stomach, suggestive of bleeding from the short gastric vessels. Splenic artery embolisation was performed with coils, which seemed to attenuate the blush. The angiogram also showed multiple beading of arteries suggestive of microaneurysms (fig 2). The relevance of this feature as a vital clue to the underlying pathology was not recognised at this stage in the course of her illness.

After a short period of about 12 hours after the angioembolisation, when she remained stable, with no further bleeding, she developed further hematemesis. A third laparotomy was undertaken. An intraoperative endoscopy was performed after a thorough open gastric wash out. The posterior wall of the fundus appeared bruised, edematous and showed a few erosions. Though there was a diffuse mild ooze of blood from the area, there was no active spurting. A total gastrectomy was carried out, with a stapled loop esophagojejunostomy.

Histology of the stomach showed necrotizing artertitis consistent with polyarteritis nodosa. She was treated with intravenous methylprednisolone, after immunological investigations, for the polyarteritis and made good initial clinical progress. However, 3 months later, she succumbed to another myocardial infarction.

Hepatitis B serology was negative. 
Senthilkumar \& Dipankar Mukherjee, "Visceral Microaneurysms of Polyarteritis Nodosa: Presentation with Recurrent Upper GI Haemorrhage needing Total Gastrectomy"

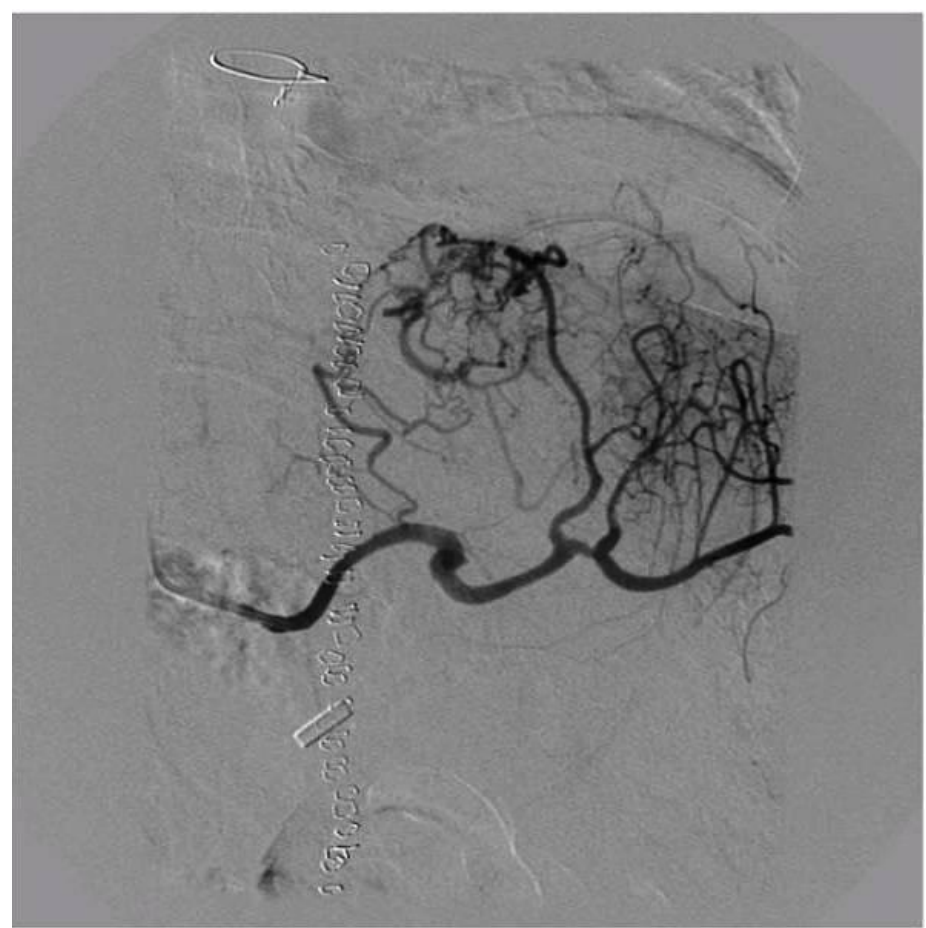

Fig2. Coeliac angiography showing microaneurysms in the splenic and gastric vascular bed.

\section{DISCUSSION}

Kussmaul and Maier described Polyarteritis nodosa in 1866. While the renal vasculature is commonly affected and the GI tract is involved in about two thirds of the patients, the pulmonary vasculature is usually spared. The small bowel is the most commonly involved part of the GIT. GI haemorrhage occurs in about $6 \%$, perforation in $5 \%$ and infarction in $1.6 \%$ of patients with PAN ${ }^{3-7}$.

Short of histopathology, there is no single diagnostic test for PAN. The American college of rheumatology has laid down ten criteria to aid the diagnosis of PAN. Presence of three out of these ten qualifies for a positive diagnosis (Table 1).

Table1. American College of Rheumatology criteria for diagnosis of PAN

\begin{tabular}{|l|l|}
\hline \multicolumn{1}{|c|}{ Criterion } & \multicolumn{1}{|c|}{ Whether present in our patient } \\
\hline Unexplained Weight loss $>4 \mathrm{~kg}$ & Yes \\
\hline Microaneurysms on angiogram & Yes \\
\hline Necrotising arteritis on histology (polymorphonuclear) & Yes \\
\hline Neuropathy & Yes \\
\hline Myalgia/ tenderness/ weakness involving lower limb & Yes \\
\hline Livedoreticularis & May be \\
\hline Elevated creatinine/ Blood urea & No baseline elevation at initial presentation \\
\hline Diastolic BP >90 & No But patient had GI bleed \\
\hline Hepatitis B positivity & No \\
\hline Testicular pain/ tenderness & Not applicable \\
\hline
\end{tabular}

Many of the symptoms that our patient had before her presentation with catastrophic upper GI bleed, including weight loss, fatigue, diarrhoea, abdominal discomfort, may be explained by PAN. She also had evidence of neuropathy involving the lower limbs and an erythematous rash in her left leg, which retrospectively are in keeping with a diagnosis of PAN. It is also tempting to speculate that the multiple myocardial infarctions she had were probably due to vasculitic lesions involving the coronary vasculature.

The Dieulafoy lesion (also known as the calibre persistent artery), is a prominent exposed submucosal vessel, which occurs with higher frequency in $\mathrm{PAN}^{8}$. Visceral microaneurysms, involving medium and small arteries, which may take the form of beads or sausages are also a classical feature of $\mathrm{PAN}^{1 .}$ However, it is important to note that microaneurysms may not be seen when angiography is performed in a hypotensive patient. 
Senthilkumar \& Dipankar Mukherjee, "Visceral Microaneurysms of Polyarteritis Nodosa: Presentation with Recurrent Upper GI Haemorrhage needing Total Gastrectomy"

In summary, though in retrospect, our patient did manifest a number of features of PAN, the acute nature of her catastrophic GI haemorrhage and the relentless recurrence of bleeding meant that the arrest of bleeding was the immediate clinical priority, overshadowing her other systemic symptoms. It is debatable what effect this 'missing the woods for the tree' phenomenon had on the clinical course of our patient. It is theoretically possible that an early diagnosis of the underlying PAN, could have allowed for a trial of steroids and immunosuppressants (such as cyclophosphamide), obviating the need for radical emergency surgery. Practically, the earliest that this could have been made in our patient, with some confidence, was after the angiogram. However, it is doubtful if medical therapy would have had its effect quick enough, as our patient did develop significant bleeding within hours of the angiogram. It may have mattered in less acute forms of bleeding.

A vasculitic process such as PAN is rarely suspected initially in the course of investigation or treatment of patients presenting with upper gastrointestinal bleeding. In patients with upper GI bleeding, maintaining a heightened awareness to the possibility of vasculitis, in the appropriate clinical context is important. These include patients with a known connective tissue or autoimmune disease; who have a history of unexplained weight loss and abdominal pain; who have a history of myocardial infarction or cerebrovascular accidents at a young age; who present with a nephritic clinical picture or skin rash or headache or fever; or who are known to be hepatitis B or HIV positive. This explicit attention to detail at initial history taking and examination is essential especially because, upper GI bleeding should always be interpreted in the context of the patient's co-morbidity and medication use. Equally crucial is the search for alternative diagnoses when none of the common causes of upper GI bleeding such as peptic ulcer, varices, erosions, esophagitis and neoplasia are discovered during the course of evaluation. A high index of suspicion for an underlying vasculitis should also be maintained if unusual findings such as a Dieulafoy lesion on endoscopy or microaneurysms on angiography are seen.

\section{REFERENCES}

[1] Stanson A, Friese JL, Johnson M,. McKusick MA, Breen JF, Sabater EA, Andrews JC Polyarteritis nodosa: spectrum of angiographic findings RadioGraphics 2001; 21:151-159.

[2] Colton CL, Butler TJ. The surgical problem of polyarteritis nodosa Br J Surg 1967; 54 (5): 393 6.

[3] SC Cooper, SC Oliff, I McCaffetery, S Wigmore, D Mirza. Polyateritis nodosa- Presenting as life threatening gastrointestinal haemorrhage. Liver transpl 14: 151-154 2008

[4] Nuzum LJW Jr, Nuzum JW. Polyarteritis nodosa: statistical review of one hundred seventy-five cases from the literature and report of a "typical case."Arch Intern Med 1954; 94:942-955.

[5] Cabal E, Holtz S. Polyarteritis as a cause of intestinal hemorrhage. Gastroenterology 1971; 61:99-105.

[6] Travers RL, Allison DJ, Brettle RP, Hughes G.Polyarteritis nodosa: a clinical and angiographic analysis of 17 cases. Semin Arthritis Rheum 1979; 8:184-189.

[7] Mowrey FH, Lundberg EA. The clinical manifestations of essential polyarteritis nodosa (periarteritis nodosa), with emphasis on the hepatic manifestations. Ann Intern Med 1954; 40:1145-1164.

[8] Ohara T, Kanoh Y, Hashimoto H. High incidence of Dieulafoy lesion in upper GI bleedingClinical examination of polyarteritis nodosa patients with rapidly progressive glomerulonephritis. Hepatogastroenterology 2008; 55(84):821-5.

Citation: Kumar S, Mukherjee D. Visceral Microaneurysms of Polyarteritis Nodosa: Presentation with Recurrent Upper GI Haemorrhage needing Total Gastrectomy. International Journal of Research Studies in Medical and Health Sciences. 2017;2(1):7-10.

Copyright: (C) 2017 Kumar S, Mukherjee D. This is an open-access article distributed under the terms of the Creative Commons Attribution License, which permits unrestricted use, distribution, and reproduction in any medium, provided the original author and source are credited. 